\title{
BL LACERTAE OBJECTS AS AN EVOLUTIONARY POPULATION*
}

\author{
A. Cavaliere, Astrofisica, Dip. Fisica, II Univ. Roma, Italy \\ E. Giallongo, Ist. Astronomia, Univ. Padova, Italy \\ F. Vagnetti, Ist. Astronomico, I Univ. Roma, Italy
}

If the BL Lac Objects are active nuclei with a beamed component that is dominant when directed at us, their observed luminosity function must comprise a flat faint branch: $N(L) d L \propto L^{1+1} / P_{d L}$ with $p=4.5$ (Urry and Shafer 1984). If this is flatter than the LF $N_{P}(L)$ of the parent objects at equal observed $\mathrm{L}$, then we expect the counts of BL Lacs to flatten out in turn at fluxes quite higher than the counts of the parents, even when both populations evolve strongly and uniformly with comparable timescales (Cavaliere, Giallongo and Vagnetti 1985).

Figs 1 and 2 illustrate the case when the parent population is a subset of the optically selected AGNs and QSOs, undergoing a luminosity evolution with a timescale $\tau \cong 0.2 \mathrm{H}_{\mathrm{o}}^{-1}$. A fraction $\mathrm{f} \ll 1$ of their isotropic power is assumed to be channelled into a randomly oriented beam of emitters with a bulk Lorentz $\Gamma \leqslant 4$; the adopted distribution $F(\Gamma) \propto \Gamma^{-1}$ minimizes the flattening to be demonstrated, yet retains an appreciable proportion of strongly beamed objects. The result is compared with a non-evolving population derived from the bright $\mathrm{E}$ galaxies, with a stronger boosting to compensate for the assumed absence of evolution and for the apparent weakness of any isotropic optical emission from these nuclei.

The final convergence at very low fluxes may be enhanced by differential evolution (slower at low L) in the parent population, or by a shorter lifetime of the beamed compared with the isotropic activity.

Two general conclusions ensue. A flat overall behaviour of the counts is not necessarily indicative of weak evolution: a flat LF anyhow allows a fast, early convergence. Conversely, a high degree of order (i.e., LF steeper than $\mathrm{L}^{-3.2}$ over most of the observed range) must underly the counts of the optically selected QSOs if they are in fact so steep $\{\mathrm{N}(<\mathrm{S})$ $\propto \mathrm{S}^{-2} \cdot{ }^{2}$ up to $\left.\mathrm{m}_{\mathrm{B}} \cong 19\right\}$ as indicated by present samples and photometry.

As for the BL Lacs specifically, the statistics of beamed and evolutionary sources predict optical counts flat on average, complying with the present observational bounds; but Fig 3 illustrates the prospects of eliciting their intrinsic evolutionary behaviour, from lower bounds to the counts at $m_{B} \cong 16.5$ over $s 1 / 2$ sky.

A similar prospect holds for the X-ray selected BL Lacs, with the expected improvement of their statistics over the bounds set from the pub1 ished MSS (Maccacaro et a1 1984).

* Discussion on p.513 


\section{REFERENCES}

Cavaliere,A., Giallongo,E., Vagnetti, F. 1985, Astr.Ap., in press (CGV). Cheng,F.Z. et al. 1985, M.N.R.A.S. 212, 857 .

Maccacaro,T. et al. 1984, Ap.J. (Letters) 284, L23.

Setti,G., and Woltjer,L. 1982, in 'Astrophysical Cosmology', Pont.Acad.

Scientiarum Scripta Varia 48, 315.

Urry,C.M. 1984, Ph.D. thesis, Johns Hopkins University.

Urry,C.M. and Shafer,R.A. 1984, Ap.J. 280, 569.

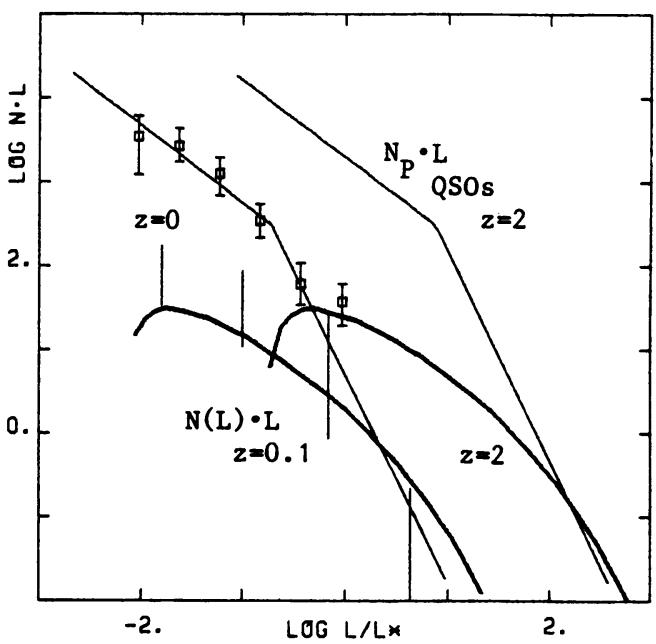

FIG.1. z-dependent LFs: Optical AGNs (1ocal data from Cheng et a1. 1985, $\tau=0.17 \mathrm{H}^{-1}$ ). Evolutionary BL Lacertae Objects derived from the former, boosted with parameters: $\Gamma$ ranging from 1 to 4 , distributed $\propto \Gamma^{-1} ; \mathrm{f}=$ $10^{-2}$; normalization to $7 \%$ of the AGNs. Bars reproduce estimates of the BL Lac LF (Urry 1984). $\mathrm{H}_{\mathrm{o}}=50 \mathrm{~km} / \mathrm{s} \mathrm{Mpc}, \Omega_{\mathrm{o}}=0.2, \mathrm{~L}^{*}=10^{30} \mathrm{erg} / \mathrm{s} \mathrm{Hz}$.

FIG.2. Predicted optical counts for evolutionary and non-evolutionary BL Lacs. Ev. BL Lacs are given in Fig 1. Non-ev. BL Lacs derive from a population constituted by $5 \%$ of the bright E galaxies, boosted with $\Gamma=7$ and $f=510^{-3}$. Observed count (square) and upper limit (triangle) from Setti and Woltjer 1982. Also given are the counts of the evolutionary parent AGNs, from the LF in Fig.1 (cf CGV 1985).

FIG.3. The BL Lac counts of Fig.2 are given in differential, normalized ( $\times \mathrm{S}^{2} .5$ ) form. Bars give $1 \sigma$ statistical uncertainties expected over $1 / 2$ sky.
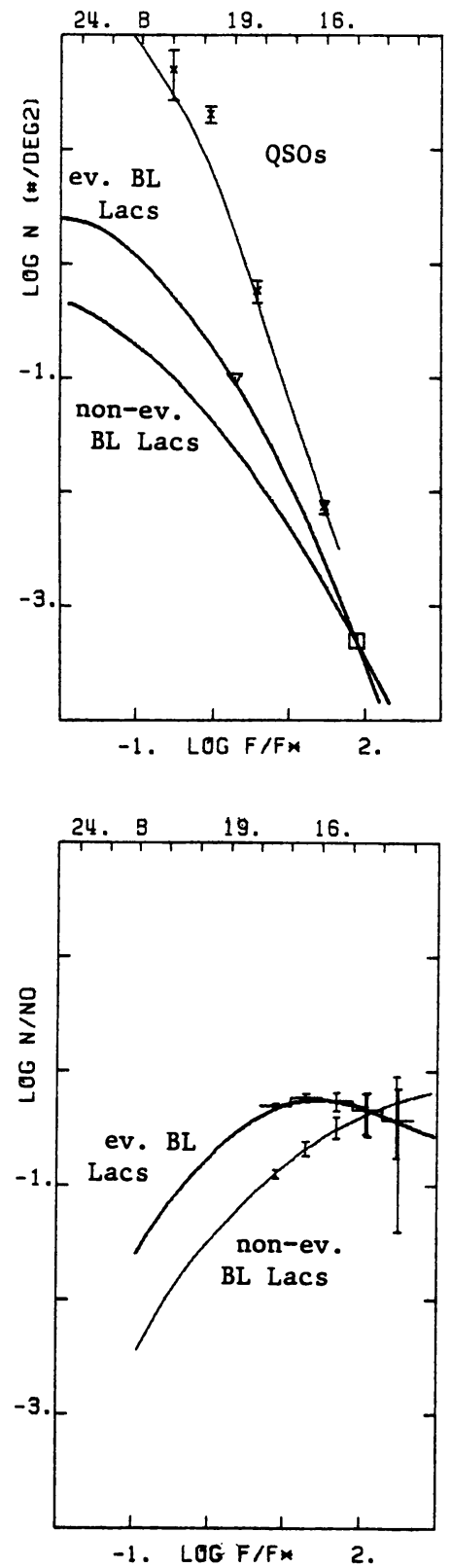